
\title{
ZS Research Square \\ WheatQTLdb V2.0: A Supplement to the Database for Wheat QTL
}

\section{Kalpana Singh}

Chaudhary Charan Singh University

Dinesh Kumar Saini

Punjab Agricultural University

\section{Gautam Saripalli}

Chaudhary Charan Singh University

Ritu Batra

Chaudhary Charan Singh University

\section{Tinku Gautam}

Chaudhary Charan Singh University

\section{Rakhi Singh}

Chaudhary Charan Singh University

\section{Sunita Pal}

Chaudhary Charan Singh University

\section{Manoj Kumar}

Chaudhary Charan Singh University

Iffat Jan

Chaudhary Charan Singh University

\section{Sahadev Singh}

Chaudhary Charan Singh University

\section{Anuj Kumar}

Chaudhary Charan Singh University

Hemant Sharma

Chaudhary Charan Singh University

Jyoti Chaudhary

Chaudhary Charan Singh University

\section{Kuldeep Kumar}

Chaudhary Charan Singh University

\section{Sourabh Kumar}

Chaudhary Charan Singh University

\section{Vikas Kumar Singh}

Chaudhary Charan Singh University

Vivudh Pratap Singh 
Chaudhary Charan Singh University

Deepak Kumar

Chaudhary Charan Singh University

\section{Shiveta Sharma}

Chaudhary Charan Singh University

\section{Sachin Kumar}

Chaudhary Charan Singh University

\section{Rahul Kumar}

Chaudhary Charan Singh University

\section{Shailendra Sharma}

Chaudhary Charan Singh University

\section{Shailendra Singh Gaurav}

Chaudhary Charan Singh University

\section{Pradeep Kumar Sharma}

Chaudhary Charan Singh University

\section{Harindra Singh Balyan}

Chaudhary Charan Singh University

Pushpendra Kumar Gupta ( pkgupta36@gmail.com )

Chaudhary Charan Singh University https://orcid.org/0000-0001-6374-9148

\section{Short Report}

Keywords: QTL, Epistatic QTL, metaQTL, Wheat, QTL database

Posted Date: February 24th, 2022

DOI: https://doi.org/10.21203/rs.3.rs-1379009/v1

License: (c) (i) This work is licensed under a Creative Commons Attribution 4.0 International License.

Read Full License 


\section{Abstract}

We recently developed a database for hexaploid wheat QTL (WheatQTLdb; www.wheatqtldb.net) that included 11,552 QTL affecting various traits of economic importance. However, that database did not include valuable QTL from other wheat species and/or progenitors of bread wheat. Therefore, an updated and wider version of wheat QTL database (WheatQTLdb V2.0) was developed in this study which now includes information on hexaploid wheat (Triticum aestivum) as well as seven other related species: $T$. durum, T. turgidum, T. dicoccoides, T. dicoccum, T. monococcum, T. boeoticum, and Aegilops tauschii. WheatQTLdb V2.0 includes a much improved list of QTL, so that V2.0 now has 27,518 main-effect QTL, 202 epistatic QTL, and 1,321 metaQTL. This newly released WheatQTLdb V2.0 will provide plant breeders and geneticists much more valuable options to search and choose the category-wise and trait-wise data for their research or breeding programmes.

\section{Introduction}

A database for wheat QTL (WheatQTLdb V1.0; www.wheatqtldb.net) was recently developed by us (Singh et al. 2021a), which contained 11,552 QTL. A correction to this database was also published later (Singh et al. 2021b). The database included QTL reported in the literature till May 2020 and included QTL for only hexaploid wheat that were identified using interval mapping and genome-wide association studies (GWAS). For the purpose of this database, marker-trait associations (MTAs) detected using GWAS were also treated as QTL. The database included not only main effect QTL, but also epistatic QTL (107) and metaQTL. This database (V1.0) proved useful which is evident from 10 citations and 824 accesses to the article received during the last seven months only. The database is also being utilized on a regular basis for improvement of the widely used alignment system Pretzel Wheat (https://plantinformatics.io/), which is an interactive, web-based environment for navigating multi-dimensional wheat datasets, including genetic maps and chromosome-scale physical assemblies (Personal Communication, Rudi Appels, University of Melbourne, Australia).

After the acceptance of our WheatQTLdb V1.0 manuscript for publication in highly conspicuous journal, Molecular Genetics and Genomics, we have been continuously working on mining and manual curation of additional QTL reported either before or after May 2020. The purpose of this manuscript is to provide updated WheatQTLdb V2.0 including information not only on T. aestivum but also on the following seven other related species: T. durum, T. turgidum, T. dicoccoides, T. dicoccum, T. monococcum, T. boeoticum, and Aegilops tauschii. WheatQTLdb V2.0 includes a much improved list of QTL, so that V2.0 now has 27,518 main-effect QTL (25,422 of T. aestivum and 2,096 of other related species), 202 epistatic QTL (162 of T. aestivum and 40 of T. durum) and 1,321 metaQTL (1,236 of T. aestivum and 85 of T. durum). This newly released V2.0 will provide users more options to search and choose the species-wise and traitwise data. We hope that this improved version of wheat QTL will prove useful to the community of wheat researchers. 
In WheatQTLdb V1.0, the traits were classified first into broad categories (e.g., biotic stresses and abiotic stresses), and then individual traits were described in each broad category. This pattern of classification of traits has been retained, while adding more QTL. The data on main-effect QTL, epistatic QTL and metaQTL have also been updated. No separate category of multi-trait QTL has been created and, wherever available, multi-trait QTL have been included in individual traits. Thus, during the present study, information for about 15,966 new main effect QTL, 95 epistatic QTL and 991 metaQTL were extracted and curated to improve the list of QTL available in WheatQTLdb V1.0. The database largely includes main-effect QTL, which make 95\% of the total QTL; the remaining 5\% QTL including metaQTL ( 4.0\%) and epistatic QTL ( 1.0\%). The species-wise distribution of different types of QTL including maineffect QTL, epistatic QTL and metaQTL are provided in Table 1, Fig. 1 and Table 2, respectively.

Table 1

Distribution of three different types of QTL in T. aestivum and other wheat species in WheatQTLdb V1.0 (Singh et al. 2021a, b) and V2.0.

\begin{tabular}{|lllll|}
\hline Types of QTL & V1.0 & V2.0 & & \\
\cline { 2 - 5 } & T. aestivum & $\begin{array}{l}\text { T. } \\
\text { aestivum }\end{array}$ & $\begin{array}{l}\text { Other } \\
\text { species }\end{array}$ & Total \\
\hline $\begin{array}{l}\text { Main-effect } \\
\text { QTL }\end{array}$ & $\begin{array}{l}11,552 \text { (25 removed due to } \\
\text { redundancy) }\end{array}$ & 25,422 & 2,096 & $\begin{array}{l}27,518 \\
(95 \%)\end{array}$ \\
\hline Epistatic QTL & 107 & 162 & 40 & $202(1 \%)$ \\
\hline metaQTL & 330 & 1236 & 85 & $1,321(4 \%)$ \\
\hline
\end{tabular}


Table 2

Frequencies of QTL for each trait belonging to nine categories of traits available in WheatQTLdb V1.0 (Singh et al., 2021a, b) and V2.0.

\begin{tabular}{|c|c|c|c|c|c|}
\hline \multirow[t]{2}{*}{ Trait category } & \multirow[t]{2}{*}{ Trait } & \multirow{2}{*}{$\begin{array}{l}\text { V1.0 } \\
\text { T. } \\
\text { aestivum }\end{array}$} & \multicolumn{3}{|l|}{ V2.0 } \\
\hline & & & $\begin{array}{l}\text { T. } \\
\text { aestivum }\end{array}$ & $\begin{array}{l}\text { Other } \\
\text { species }\end{array}$ & Total \\
\hline \multirow[t]{3}{*}{$\begin{array}{l}\text { Nutrient use } \\
\text { efficiency }\end{array}$} & $\begin{array}{l}\text { Phosphorus use } \\
\text { efficiency }\end{array}$ & 185 & 236 & 0 & 236 \\
\hline & Nitrogen use efficiency & 1,338 & 1,702 & 0 & 1,702 \\
\hline & $\begin{array}{l}\text { Potassium use } \\
\text { efficiency }\end{array}$ & 745 & 1,049 & 0 & 1,049 \\
\hline \multirow[t]{5}{*}{ Abiotic stress } & Water logging tolerance & 282 & 277 & 0 & 277 \\
\hline & Salt stress tolerance & 687 & 1,603 & 58 & 1,661 \\
\hline & Heat stress tolerance & 453 & 1,812 & 396 & 2,208 \\
\hline & $\begin{array}{l}\text { Pre-harvest } \\
\text { sprouting tolerance }\end{array}$ & 593 & 574 & 25 & 599 \\
\hline & Drought tolerance & 1,414 & 1,685 & 383 & 2,068 \\
\hline \multirow[t]{4}{*}{ Biotic stress } & Nematode resistance & 85 & 162 & 02 & 164 \\
\hline & Insect resistance & 111 & 150 & 0 & 150 \\
\hline & Bacterial resistance & 24 & 29 & 0 & 29 \\
\hline & Fungal resistance & 3,007 & 3,421 & 285 & 3,706 \\
\hline \multirow[t]{3}{*}{ Biofortification } & Zinc content & 449 & 526 & 15 & 541 \\
\hline & Selenium content & 71 & 62 & 15 & 77 \\
\hline & Iron content & 312 & 367 & 25 & 392 \\
\hline Yield & Yield & 712 & 6,863 & 651 & 7,514 \\
\hline Developmental & Developmental & 56 & 779 & 89 & 868 \\
\hline Physiological & Physiological & 391 & 427 & 0 & 427 \\
\hline Morphological & Morphological & 153 & 951 & 61 & 1,012 \\
\hline Quality & Quality & 484 & 2747 & 91 & 2,838 \\
\hline Total & & 11,552 & 25,422 & 2,096 & 27,518 \\
\hline
\end{tabular}

\section{Data Summary And Availability}


The QTL data available in V1.0 and V2.0 are summarized in Table 1. It can be seen that the number of main-effect QTL and metaQTL in V2.0 is more than double in comparison to corresponding numbers in V1.0. The current version 2 of wheat QTL database is available at http://wheatqtldb.net and has no access restriction to academic use of the database.

\section{Main-effect QtI For Different Traits}

In WheatQTLdb V2.0, now there are 27,518 QTL, of which 25,422 QTL belong to T. aestivum and the remaining 2,096 QTL belong to other seven wheat species. The distribution of QTL in other species (Fig. 1 ) is as follows: T. durum (2,055), T. monococcum (02), T. boeoticum (05), T. turgidum (11), T. dicoccoides (09), T. dicoccum (05) and Aegilops tauschii (09). These QTL belong to 21 different traits placed in nine major categories (Table 2). The maximum number of QTL are available for yield followed by abiotic and biotic stresses. Figure 2 shows chromosome-wise distribution of QTL in hexaploid wheat and related species; it is apparent that B sub-genome has maximum percentage of QTL (40.78\%), followed by A sub-genome (37.66\%) and D sub-genome (21.54\%) in that order. Chromosome $2 \mathrm{~B}$ carries the maximum number of QTL followed by chromosomes 1B, 5A and 3B. Figure 3 shows frequency of QTL in hexaploid wheat and related species according to year of publication. It can be seen that major progress in QTL discovery was made during the last 5 years, mainly due to major focus in the area of GWAS and increased activity in the area of QTL interval mapping.

\section{Metaqtl For Different Traits}

In WheatQTLdb V1.0, there were 330 metaQTL belonging to drought tolerance, heat stress tolerance, root depth and leaf rust resistance. V 2.0 has 1,321 metaQTL, of which 1,236 metaQTL belong to T. aestivum with the following traits: earliness, grain yield, protein content, heading time, iron content, zinc content, multiple disease resistance, nitrogen use efficiency, phosphorus use efficiency and plant height. The remaining 85 metaQTL belong to T. durum with the following traits: biotic stresses, abiotic stresses and quality traits. This rise in frequency of metaQTL is mainly due to several new studies on metaQTL published during 2019-2021, which is also evident from Fig. 4 showing frequencies of metaQTL according to year of publication. Figure 5 shows chromosome-wise distribution of metaQTL in $T$. aestivum and T. durum available in V2.0; it can be seen that the B sub-genome has the maximum number of metaQTL followed by $A$ and $D$ sub-genomes.

\section{Epistatic Qtl Information}

In WheatQTLdb V1.0, there were 107 epistatic QTL for T. aestivum belonging to the following traits: heat stress tolerance, insect resistance, physiological traits, quality-related traits, stem rust resistance, stripe rust resistance, and yield-related traits. V2.0 has 202 epistatic QTL, of which 162 QTL belong to $T$. aestivum pertaining to salt stress tolerance, and nitrogen use efficiency along with traits mentioned in V1.0. The remaining 40 epistatic QTL belong to $T$. durum pertaining to quality- and yield-related traits. 


\section{Enhanced Features Of Wheatqtldb V2.0}

Following updates have been made in WheatQTLdb V2.0 web-resource to improve its utility for wheat researchers: (i) Addition of more main-effect QTL and metaQTL. (ii) Updated genetic maps for QTL (according to wheat reference genome). (iii) Better search options making the web-server more userfriendly. (iv) New graphs for better representation of data in the statistics section. (v) Updated web-site for improved user experience while handling web-resource (Fig. 6 shows layout of updated web-page of WheatQTLdb V2.0).

\section{Conclusions}

A large number of main-effect QTL, epistatic QTL and metaQTL have been added to WheatQTLdb V1.0, which was already the largest collection of QTL, metaQTL and epistatic QTL in a single database for $T$. aestivum. WheatQTLdb V2.0 replaces V1.0 and provides much more inclusive data for QTL of $T$. aestivum and other seven related species, published till December, 2021. Hopefully, the V2.0 will serve better the wheat research community including research scholars, wheat geneticists and wheat breeders. The WheatQTLdb V2.0 is available at http:// wheatqtldb.net.

\section{Declarations}

\section{Acknowledgments}

Thanks are due to Chaudhary Charan Singh University, Meerut and Bioinformatics Infrastructure Facility, New Delhi for providing the necessary computational resources for the development of WheatQTLdb V2.0. Thanks are also due to Indian National Science Academy (INSA), New Delhi for the award of the positions of INSA-Senior Scientist and INSA Honorary Scientist to HSB. Thanks are also due to Department of Biotechnology (DBT), Govt. of India for awarding research projects to PKS and SS, including RLS scheme to SS.

\section{Author's contributions}

PKG, HSB, PKS, SSG, SS (Shailendra Sharma), RK and SK (Sachin Kumar) conceived and planned the study and also finalized the manuscript. KS prepared the database and helped DKS and GS in preparation of the first draft of the manuscript. All the remaining authors collected and tabulated the data for different traits. DKS prepared the chromosome maps showing the markers linked to QTLs for different traits.

\section{Funding}

This research received no external funding. 
The authors declare that they have no conflict of interest.

\section{Data availability}

Updated database (WheatQTLdb V2.0) can be accessed online from the following URL:

www.wheatqtldb.net through any internet browser (Mozilla Firefox/Chrome/Safari/Internet Explorer) compatible as per the operating system (Windows/Linux/Mac OSX).

\section{Code availability}

Not applicable

\section{Ethics approval}

Not applicable

\section{Consent to participate}

Not applicable

\section{Consent for publication}

Not applicable

\section{References}

1. Singh K, Batra R, Sharma S et al (2021a) WheatQTLdb: a QTL database for wheat. Mol Genet Genomics 296:1051-1056

2. Singh K, Batra R, Sharma $S$ et al (2021b) Correction to: WheatQTLdb: a QTL database for wheat. Mol Genet Genomics 296:1359-1360. https://doi.org/10.1007/s00438-021-01812-y

\section{Figures}




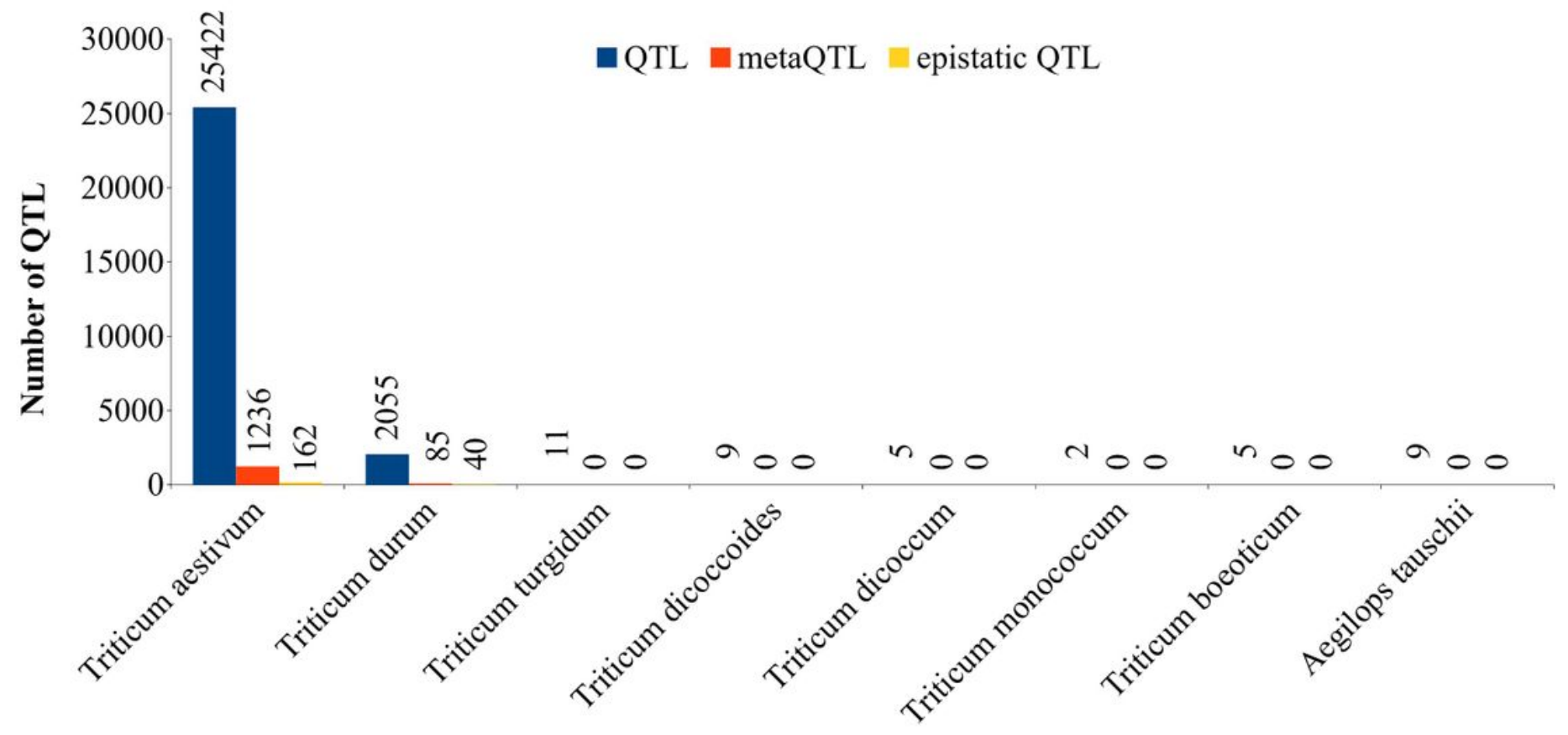

Figure 1

Species-wise distribution of main-effect QTL, metaQTL, and epistatic QTL in WheatQTLdb V2.0

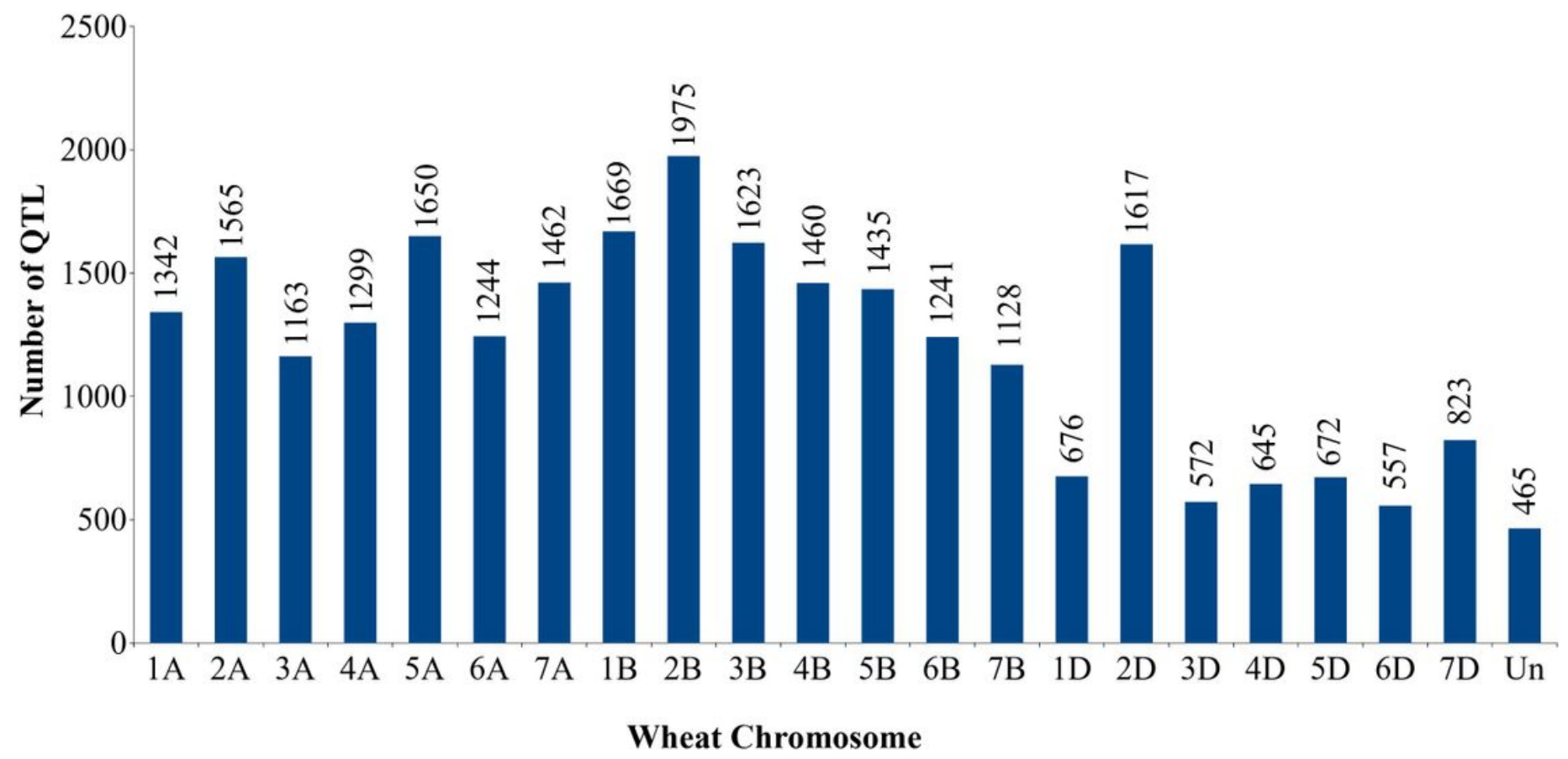

Figure 2

Chromosome-wise frequencies of different types of QTL in WheatQTLdb V2.0 


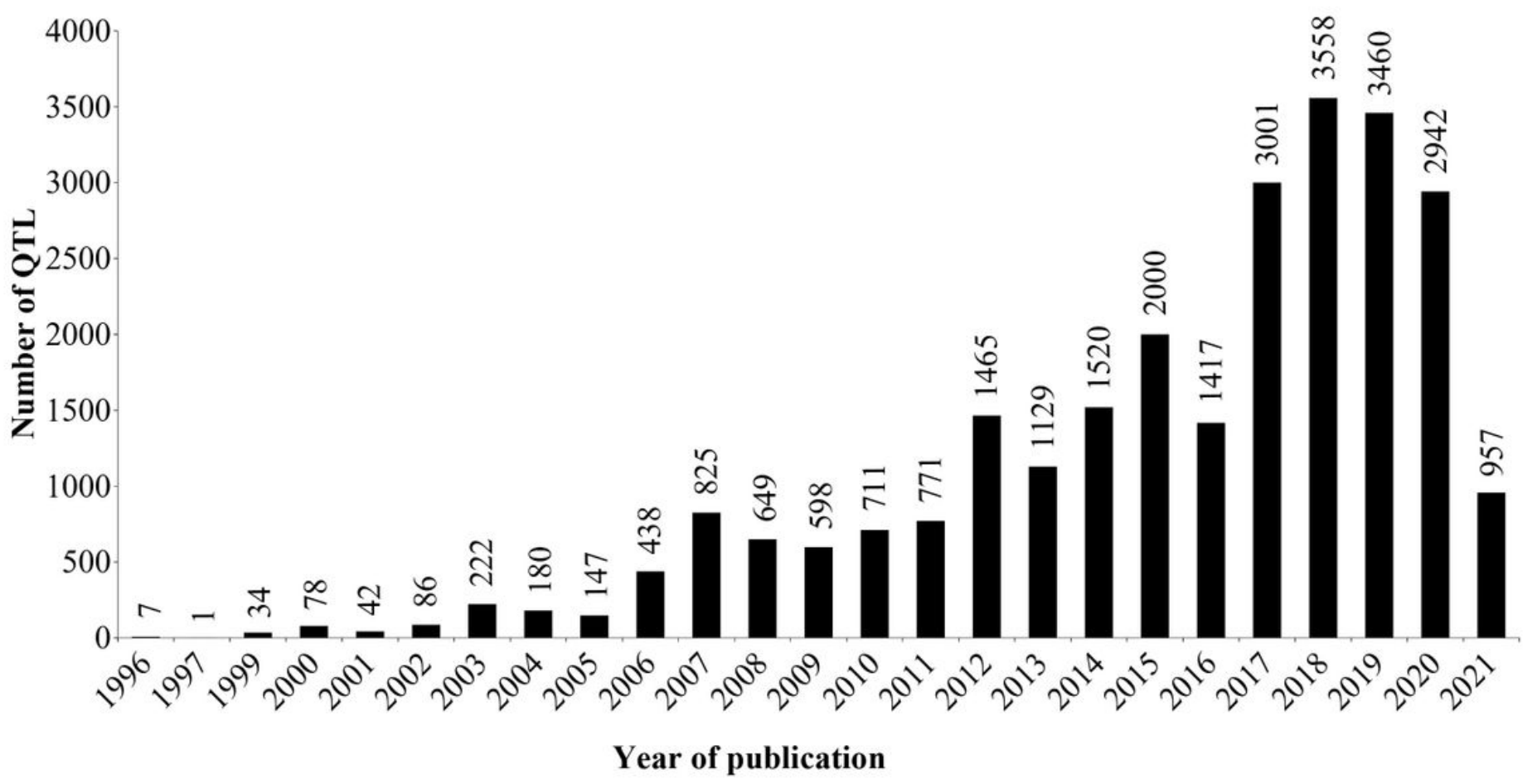

Figure 3

Frequencies of year-wise reported main-effect QTL in WheatQTLdb V2.0

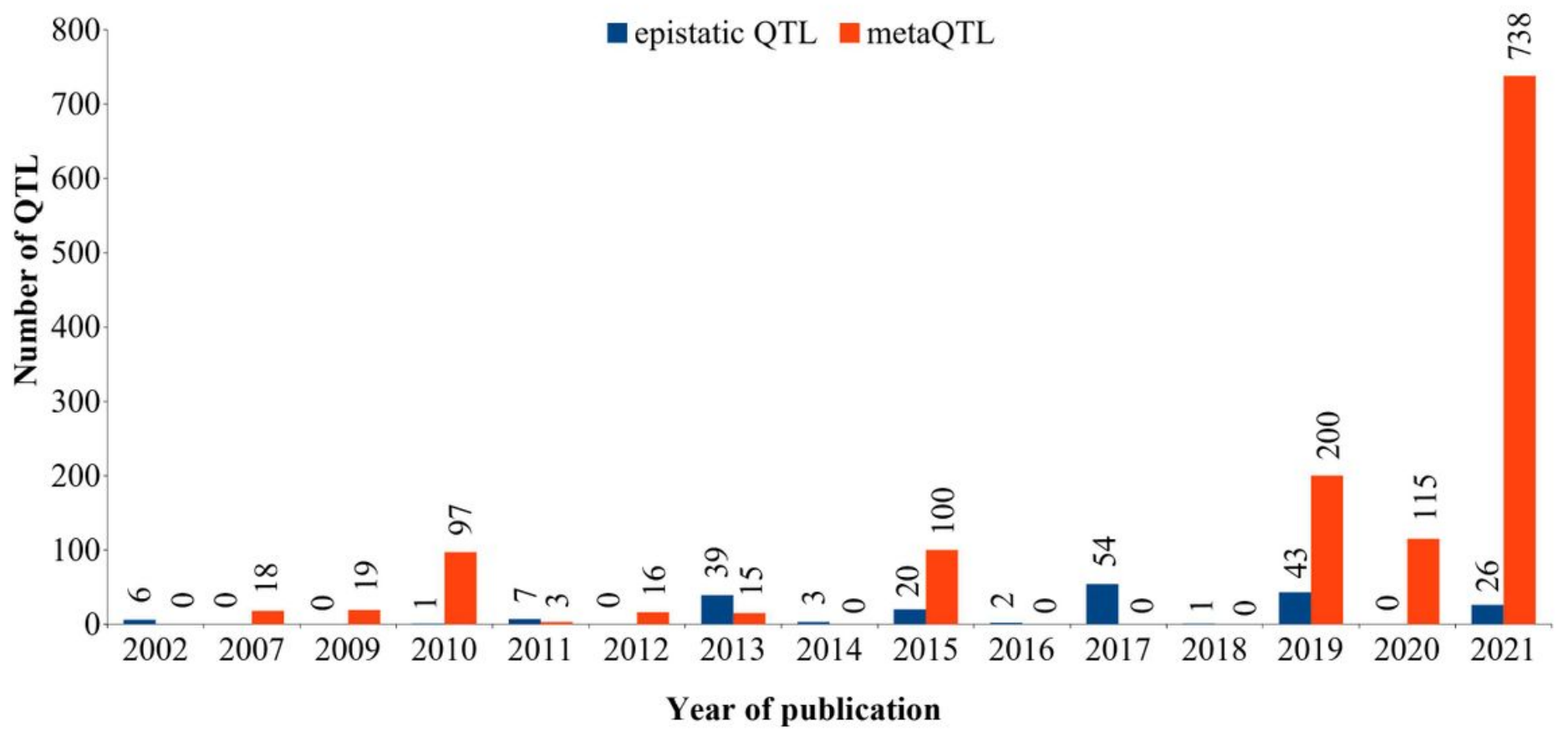

Figure 4

Frequencies of year-wise reported epistatic QTL and metaQTL in WheatQTLdb V2.0 


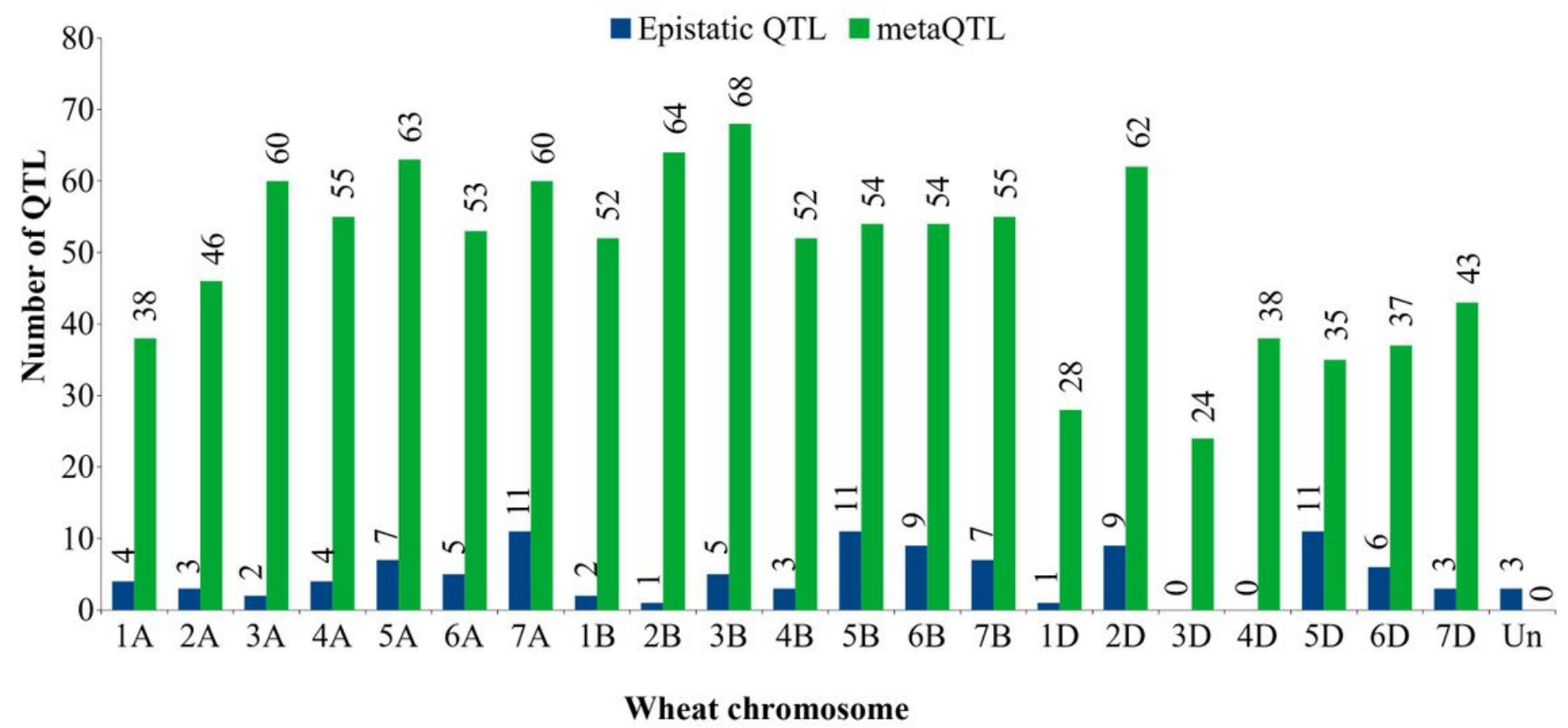

Figure 5

Chromosome-wise distribution of epistatic QTL and metaQTL in WheatQTLdb V2.0
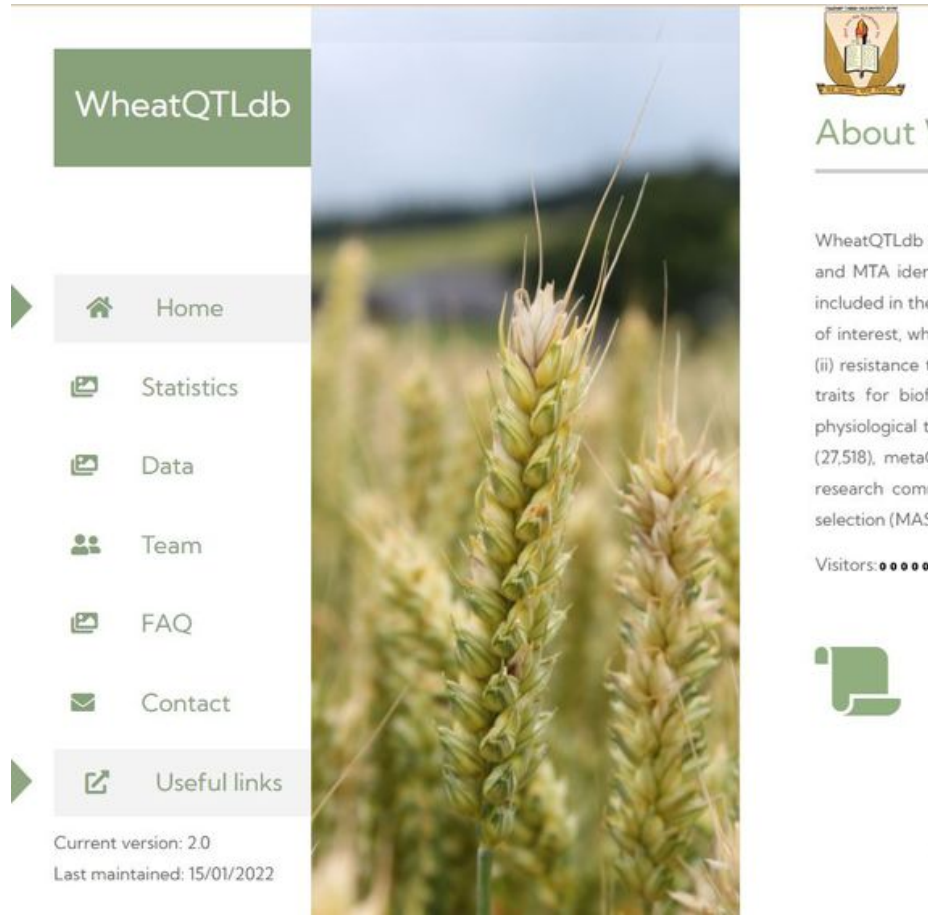

\section{About Web-Resource}

WheatQTLdb V2.0 is a manually curated QTL database for wheat that includes information about QTL identified through interval mapping and MTA identified using GWAS. The available information on metaQTL, epistatic QTL and candidate genes, wherever available, is also included in the database. Users could browse and download the database to find information regarding the genetic architecture of the traits of interest, which include the following: (i) tolerance to abiotic stresses including drought/water logging/heat/pre-harvest sprouting/salinity: (ii) resistance to biotic stresses including not only viral, bacterial and fungal diseases, but also to infestation by nematodes and insects; (iii) traits for biofortification ( $\mathrm{Fe} / \mathrm{Se} / \mathrm{Zn}$ contents): (iv) developmental traits; (v) morphological traits; (vi) N/P/K use efficiency traits; (vii) physiological traits; (viii) quality traits; and (ix) yield and its related traits. WheatQTLdb is the largest web resource having collection of QTL, (27.518), metaQTL (1,321), epistatic QTL (202) in Triticum aestivum and 07 other related wheat species to serve the international wheat research community including plant breeders and geneticists for further studies involving fine mapping, cloning and marker assisted selection (MAS) during wheat breeding

Visitors:000003170

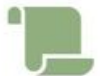

How to cite us

If you use information gathered from this site in any publication, please cite the following paper:

Singh K, Batra R, Sharma S, Saripalli G, Gautam T, Rakhi, KumarJ, Pal S, Malik P. Kumar M Lateef I, Singh S, Kumar D, Saksham, Chaturvedi D, Verma A, Rani A, Kumar A, Sharma H, Jyoti, Kumar K Kumar S, Singh VK. Singh VP, Kumar S, Kumar R. Sharma S, Gaurav SS. Sharma PK, Balyan HS, Gupta PK, 2020. WheatQTLdb: a QTL database for wheat. Mol Genet Genomics 296, 1051-1056 (2021). https://doi.org/10.1007/s00438-021-01796-9

Figure 6

Layout of updated web-page of WheatQTLdb V2.0 